AROUEOLOGÍA Y SOCIEDAD

№ 24, 2012: $479-488$

ISSN: 0254-8062

INFORME

\title{
MARCO CONCEPTUAL PARA LA CREACIÓN DEL MUSEO NACIONAL AMAZÓNICO
}

\author{
SANTIAgO Rivas PANDURO \\ COMISIÓN DE ALTO NIVEL PARA LA CREACIÓN DEL MUSEO NACIONAL AMAZÓNICO \\ DiRECCIÓN REgIONAL DE CULTURA - MiNISTERIO dE CULTURA
}

\section{Presentación}

El presente artículo es el resultado del trabajo encomendado al autor para elaborar el Marco Conceptual que sustente la necesidad de la creación de un Museo Amazónico en la ciudad de Iquitos, promovida por el Congresista de la República por Loreto, Dr. Víctor Isla Rojas.

En efecto, el 04 de abril de 2012 el Dr. Isla invitó a un grupo de investigadores amazónicos a una reunión de trabajo en Lima, en el Congreso, con el fin de tratar e intercambiar ideas en torno al tema: "Desarrollo cultural de la región Loreto con miras a la creación de un Gran Museo Amazónico". En ella participaron el propio congresista Víctor Isla Rojas, Maritza Ramírez Tamani (educadora), Marcos Celis (economista), Francisco Grippa Jochamowitz (artista plástico), Gino Ceccarelli Bardales (artista plástico), Jaime Vásquez Valcárcel (Editorial Tierra Nueva), Francisco Bardales (abogado y escritor), Joaquín García Sánchez (investigador - Director del Centro de Estudios Teológicos de la Amazonía y gestor cultural), José Álvarez Alonso (investigador ambiental - biólogo), José Alberto Chirif Tirado (investigador social - antropólogo), Róger Rumrill García (investigador - periodista), Jean Pierre Chaumeil (investigador social - antropólogo), Santiago Rivas Panduro (investigador social - arqueólogo), Martín Reátegui Bartra (investigador social - educador), Daniel Gabel Sotil García (investigador social - educador), James Matos Tuesta (investigador social - abogado - periodista), y Gustavo Rodríguez (publicista).

Los acuerdos de esta importante reunión fueron:

- Conformar una comisión de alto nivel que participe en la elaboración del marco conceptual para la creación del Museo Amazónico en Iquitos y la elaboración del término de referencia para la consultoría del proyecto del Museo Amazónico en Iquitos, la misma que estuvo conformada por los participantes a la precitada reunión.

- Encargar dicha tarea a Santiago Rivas Panduro, en un plazo de un mes.

Las fuentes que sustentaron la elaboración de este Marco Conceptual fueron:

- Los aportes de los miembros de la comisión de alto nivel, durante la reunión del 4 de abril, sintetizados en el concepto global de un museo dinámico, de espacio abierto, interactivo, social y ambiental. 
- La revisión bibliográfica, citadas en el presente documento.

- Los comentarios, observaciones, críticas, y bibliografía facilitada por los miembros de la comisión de alto nivel.

- Los aportes de colegas especialistas en patrimonio cultural, patrimonio natural, Arc Gis e imágenes satelitales.

Este marco conceptual fue presentado al congresista Isla Rojas el 31 de mayo de 2012. A la fecha. 5 de setiembre de 2012, el Ministerio de Cultura viene desarrollando los procedimientos formales para los estudios del Proyecto del Museo Nacional Amazónico, y de su parte el Dr. Isla Rojas viene gestionando el Convenio entre el Ministerio de Cultura y la Universidad Nacional Mayor de San Marcos, nuestra Alma Mater, para el otorgamiento del terreno para el Museo.

Cabe resaltar además, que durante la celebración de entrega del galardón del Río Amazonas Maravilla Natural del Mundo, en agosto último, Iquitos recibió con beneplácito el anuncio de su realización, por el presidente de la república, comandante Ollanta Humala Tasso.

\section{ANTECEDENTES}

La región amazónica no cuenta con infraestructura propia de un museo, la que estaría ubicada en la urbe cosmopolita más importante de la Amazonía peruana, Iquitos, la sexta ciudad más poblada del Perú con 452.757 habitantes, de acuerdo al censo del INEI 2007. Iquitos no tuvo una fundación como pueblo o ciudad, pero sí como puerto fluvial fechado en 5 de enero de 1864, marcado con la llegada de los barcos de vapor que mandara a construir el presidente Ramón Castilla. Pero la historia de Iquitos se relaciona con el pueblo misional San Pablo de Nuevo Napeanos, reducido por el padre Jesuita Bahamonde hacia 1761, con indígenas mayoritariamente Iquitos y Napeanos, ubicado por aquel entonces en la planicie alta que ocupa esta ciudad (Sotil 2002). Hacia 1835, durante el gobierno del presidente de la república del Perú, Luis José Orbegoso, quien protegió la expedición inglesa de Smyth y Lowe, se tiene información de la existencia del pueblo Santa Bárbara de Iquitos, localizado en la ribera norte del Amazonas, cerca al río Momón, a la sazón pueblo pequeño con 60 habitantes mestizos "más o menos"; para esas fechas los Iquitos ya se habían retirado aguas arriba del Nanay (Espinoza 2007: 533-8).

Desde varias décadas atrás hasta hace algunos años en Iquitos había hasta cinco "museos", ninguno con local propio. Existió desde la época del Mons. García Pulgar, un pequeño museo de los bienes de la cultura material de las poblaciones, pertenecientes al Vicariato Apostólico de Iquitos; este museo funcionó en la casa antigua del Vicariato. En otro momento no tan lejano al anterior, con ocasión del Centenario de Iquitos como Capital de Loreto, durante el periodo del comandante general EP Gral. José Benavides, se instaló un museo a cargo del Ejército, en una vivienda casona de la calle Próspero.

Estaba también el Museo Municipal denominado también Museo de Ciencias Naturales, de la municipalidad provincial de Maynas, creado en 1974. Este museo cambió de local repetidas veces, y feneció en 2003, junto con la exhibición descuartizada y en malísimo estado de conservación de especies disecadas de nuestra fauna acuática, terrestre, arbórea y aérea. Teníamos también la sala de exposición de suelos amazónicos del Centro de Referencia de Información de Suelos de la Amazonía Peruana (CRISAP), denominado también Museo de Suelos de la Universidad Nacional de la Amazonía Peruana (UNAP), inaugurado en 1994 con una exhibición de muestras de suelos minerales amazónicos, que no logró renovarse con los años venideros, al punto que por diversas razones fue cerrado por una temporada en la primera década del presente siglo, aunque hoy siguen exhibiéndose las columnas de suelo estabilizada con resinas sintéticas en el mismo ambiente donde también se usa como aula para las clases sobre suelos amazónicos para los alumnos de agronomía, forestal y biología de la UNAP.

Luego está el llamado Museo Amazónico, inaugurado en 1994 por el entonces prefecto de Loreto y a la vez presidente del Consejo Transitorio de Administración Regional de Loreto, Tomás Gonzales 
Reátegui, del cual sólo quedan en exhibición en los ambientes de la hoy gobernación de Loreto, algunos cuadros contemporáneos despintados, y una decena de esculturas de Felipe Lettersten, de indígenas amazónicos en fibra de vidrio, actualmente rotas y quebradas.

Además, a falta de museo propiamente, en Iquitos y Yurimaguas hubo varias exposiciones museográficas, con la finalidad de resaltar y dar a conocer los valores culturales amazónicos. Una de ellas fue la exposición de cerámica amazónica en el Centro Internacional en la Plaza de Armas, en el marco del IV Festival Internacional de la Canción Amazónica (FICA), en 1982. Años más tarde, el 2004 también se abrió una sala de exposición arqueológica amazónica, temporal, en los ambientes del Museo Amazónico de Iquitos, en los bajos de la actual gobernación, dentro de la programación de la semana turística de Iquitos. También, en Iquitos se han desarrollado dos importantes exposiciones museográficas, Cosmovisiones Amazónicas (2001) y La Serpiente de Agua (2003), ambas organizadas por el Programa de Formación de Maestros Bilingües ejecutado por AIDESEP en convenio con el Ministerio de Educación.

Igualmente, en Yurimaguas, se realizó la I muestra arqueológica de la provincia de Alto Amazonas, organizado por la gerencia subregional de Alto Amazonas, que además de instalarse en un local del centro de la ciudad, se desplazó a una institución educativa del nivel secundario para que los alumnos puedan tener acceso directo a la muestra.

Es de necesidad contar con un museo apropiado para la Amazonía peruana que tenga mucha llegada a gente, y no seguir estando a espaldas del país o viceversa, en este escenario cultural; sino antes bien, ser protagonistas de los procesos generadores de identidades, creatividad, educabilidad, logros y posibilidades de desarrollo, a la vez que sea el soporte para la integración, valorización, fortalecimiento y consolidación de esta región del país con la del resto del Perú.

Sintiendo esta necesidad, en los últimos años hubo varias propuestas de creación, equipamiento y funcionamiento de museos para la región Loreto, pero ninguna de ellas se concretó hasta la fecha. Así tenemos:

1. La primera propuesta escrita que disponemos es "Amazonium: memorial de la biodiversidad y del hombre en la Amazonía”, propuesto por el Consorcio Amazonium, conformado por el Centro Amazónico para el Desarrollo Sostenible, la Universidad Nacional de la Amazonía Peruana, y el Centro de Estudios Teológicos de la Amazonía, cuyo documento fuera publicado en 2001.

2. La Ordenanza Regional N 015-GRL/CR, del 17 de julio de 2003, mediante el cual ordena: artículo primero: Declarar "Capital Arqueológica de la Región Loreto a la provincia de Alto Amazonas"; artículo segundo: Impulsar la Creación de un Museo Arqueológico el cual funcione de manera transitoria en los ambientes de la Sub Región de Alto Amazonas. El 2006, cumpliendo con la precitada Ordenanza, la Gerencia Sub Regional de Alto Amazonas elaboró el perfil del proyecto "Mejora y Equipamiento del local de la Gerencia Sub Regional de Alto Amazonas para el Museo Arqueológico y Etnográfico de Yurimaguas”, con una inversión de 722,656.45 Nuevos Soles.

3. El Proyecto de Inversión Pública "Acondicionamiento del Parque Turístico Nacional Laguna Quistococha, en el distrito de San Juan Bautista, provincia de Maynas - región Loreto" - Código SNIP 144022 (2010), a través de la Puesta en Valor del Sitio Arqueológico Quistococha, el mismo que incluye investigaciones con excavaciones arqueológicas, y construcción, equipamiento y funcionamiento de un museo de sitio, con un costo de inversión de 2 631,910.00 Nuevos Soles. La inserción de este componente fue posible merced al soporte técnico proporcionado por el Departamento de Arqueología y Departamento de Arquitectura de la Dirección Regional de Cultura de Loreto - Ministerio de Cultura, a los consultores del Plan Copesco Nacional/MINCETUR encargados de la formulación del referido proyecto.

4. La propuesta presentada por el Director Regional de Cultura de Loreto, en marzo de 2012, al viceministro de Industrias Culturales del Ministerio de Cultura, de un "Museo de la Amazonía. Museo del Caucho". 


\section{LORETO EN EL CONTEXTO AMAZÓNICO, CULTURAL Y AMBIENTAL}

La Amazonía peruana abarca el 60,9\% del territorio nacional, que incluye la totalidad de los departamentos de Loreto, Ucayali, Madre de Dios y San Martín, y con partes de los departamentos de Cajamarca, Amazonas, La Libertad, Huánuco, Pasco, Junín, Cuzco, Puno, Huancavelica, Ayacucho, Apurímac. Según el censo del INEI 2007, su población nacional corresponde al 13,4\% (3 675292 hab.) de la población total del Perú cifrada en 28220764 habitantes; a su vez, su población indígena comprende el 9\% (332 975 hab.) de la población amazónica total (Benavides 2010: 17), aunque para el 2005 dicha cifra ascendía a 330000 indígenas amazónicos, repartidos en 59 lenguas y 15 familias lingüísticas (Instituto del Bien Común IBC 2005).

Loreto, con su selva baja y selva alta (Pulgar Vidal 1985), es la región enteramente amazónica, y la más extensa entre todos los departamentos del país, con $368851,95 \mathrm{~km}^{2}$ de territorio (Vega 2008: 34), en el cual habitan pueblos representantes de unas 10 a 13 familias lingüísticas amazónicas (Pozzi Escot 1998; Solís 2003) distribuidas en territorios indígenas, titulados y no titulados. Otros territorios se suman a los anteriores, concedidos por el Estado a terceros naturales o privados, nacionales o extranjeros, para usos diversos, como lotes de hidrocarburos, concesiones forestales y concesiones mineras, o para protección de algunos recursos inherentes en él, como los bosques de producción permanente, las áreas naturales protegidas y las áreas de conservación regional (Benavides 2010).

Pero esta distribución del territorio amazónico viene reduciendo cada vez más las tierras ancestrales de los pueblos indígenas y de sus parientes mestizos, ocasionando muchos conflictos socioambientales, por el uso y explotación irracional de los recursos y/o presión sobre los recursos por agentes externos, como también por la propia población originaria del lugar, incentivada por la economía de mercado.

Los enfrentamientos por tierras al que se ven obligado enfrentar las comunidades indígenas, aisladas o no, se dan pues con la legalidad del uso de otras tierras, que amenazan, contaminan, limitan o afectan el uso y manejo cultural de sus recursos; así tenemos a las áreas naturales protegidas o los lotes de hidrocarburos, colindantes o sobrepuestos al territorio indígena, que en muchos casos va más allá de los límites formales de tierras tituladas o no (Chirif 2010: 64, 70).

De otro lado, esta explotación irracional de la Amazonía se da de múltiples formas, desde las simples y directas hasta las sofisticadas e indirectas, con la tala y caza ilegales, o con la apropiación de los conocimientos ancestrales para la aplicación de medicamentos, respectivamente, para citar algunos casos. Pero sin el mínimo beneficio, en términos de equidad racional, para los dueños de estos conocimientos y territorios (Álvarez 2010: 39).

Las culturas amazónicas conforman también un mosaico diverso, cual reflejo de su pluralidad, complejidad y diversidad heredadas desde las culturas ancestrales que poblaron la cuenca amazónica en sucesivas oleadas migratorias, aproximadamente entre 11,000 - 9,500 antes del presente, según información consensuada (Aceituno 2010; Neves 2011), pero también por las estrategias adaptativas resultado de las migraciones y/o interrelaciones andino-costeñas, desde épocas prehispánicas, por lo menos desde hace 5 mil años hasta antes de la invasión española, con las culturas Caral, Chavín, Paracas, Nasca, Moche, Chimú (Rivas 2011), así como por las estrategias de sobrevivencia y sincretismo con el mestizaje de las recientes colonizaciones occidentales, asiáticas y africanas, en los últimos 500 años, tomadas como referencia temporal el descubrimiento europeo del estuario del Amazonas por Juan Vicente Núñez Pinzón, el 26 de enero de 1500, al decir de Rumrrill (2011). El curso propiamente dicho del río Amazonas fue reconocido por Francisco de Orellana 1542 (Carbajal 2009).

Esta herencia cultural ancestral se sintetiza en saber profundo y extenso del mundo circundante, que los pobladores amazónicos aún conservan (Rumrrill 2011).

La diversidad y complejidad de los recursos naturales existentes en Loreto, y de la Amazonía en general, es decir, de sus suelos, flora y fauna, es resultado de fenómenos y procesos diversos a lo lar- 
go de la historia geológica y biológica, como el levantamiento de la cordillera de los Andes, la deriva continental, los vientos interoceánicos, aumentos y/o disminución de temperatura y precipitación influenciados por las glaciaciones, el levantamiento o hundimiento y acumulación de los depósitos fluviales, la dinámica hidrológica y envergadura hídrica, la naturaleza y formación de sus aguas, etc. En este escenario, los principales acontecimientos que dan configuración a lo que hoy es la Amazonía peruana se remontan desde la época Neógeno que comprende el Mioceno (23-6 m.a.) y el Plioceno (6 - 2 m.a.), seguido por el Pleistoceno (2 m.a. - 10 mil años) y luego el Holoceno (10,000 hacia adelante) (Kalliola, Puhakka y Danjoy 1993; Flores Paitán 1998). Fenómenos como los friajes, los veranillos, El Niño y La Niña también son causantes de las dinámicas ambientales en nuestra Amazonía (Marengo 1998).

Es pues la región Loreto, aunque no el único, un territorio promisorio, por la belleza de los paisajes y la riqueza de sus ecosistemas, pero por sobre todo por los conocimientos que los pobladores indígenas tienen sobre la diversidad de plantas, animales, y germoplasma, útiles para la humanidad (Rumrrill 2011).

De allí la importancia de educar y valorar nuestra Amazonía, es decir nuestra naturaleza humana y nuestros recursos naturales, como principal punto de partida hacia un desarrollo socio-ambiental sostenible. Por ello también es importante formar, concientizar y cultivar en la población el respeto y protección de nuestro Patrimonio Cultural y Patrimonio Natural, que por su importancia, trascendencia y excepcionalidad, gozan de protección nacional (Ley N² 28296, Ley General del Patrimonio Cultural de la Nación; Ley $N^{\circ}$ 26834, Ley de Áreas Naturales Protegidas), e internacional (directrices prácticas para la aplicación de la Convención del Patrimonio Mundial. Comité Intergubernamental de Protección del Patrimonio Mundial cultural y natural. Centro del Patrimonio Mundial de la UNESCO. 2005. París).

\section{El Museo Nacional Amazónico, necesidad regional de perspectiva nacional}

La carencia de un Museo Nacional Amazónico, es un indicador, entre otros tantos, del atraso de nuestra región, por lo de menos 50 años en política educativa y cultural, si comparamos las fechas de creación de otros museos del país, sólo para mencionar algunos, como son:

Museos Nacionales:

- Museo Nacional de Antropología, Arqueología e Historia del Perú (1822)

- Museo Nacional de la Cultura Peruana de Lima (1946)

- Museo Nacional Chavín (2008)

Museos Regionales:

- Museo Arqueológico de Áncash (1935)

- Museo Histórico Municipal “Guillermo Zegarra Meneses” de Arequipa (1953)

- Museo Histórico Regional de Ayacucho (1943)

- Museo de Arte Religioso de Cajamarca (1965)

- Museo Regional de Ica (1947)

- Museo de Arte Salesiano de Huancayo (1973)

- Museo de Zoología "Juan Ormea" de Trujillo (1938)

- Museo Regional de Lambayeque "Enrique Bruning” (1921)

- Museo Orve de Pasco (1975)

- Museo Tumbas Reales de Sipán - Lambayeque (2002)

En el Perú, uno de los principales promotores y creadores de los más importantes museos fue el sabio y padre de la arqueología peruana Julio C. Tello (1880-1947) (Del Águila 2007: 6), pero bajo la propuesta de cumplir una función educativa, tal como lo había plasmado en su proyecto en junio de 1913 titulado "Presente y Futuro del Museo Nacional", cuando ingresó a trabajar en el Museo 
Nacional como Jefe de la Sección Arqueología, causando conflicto con el entonces director de la Sección Histórica del Museo Nacional, quien mantenía la tesis que el museo era un lugar de "disfrute para los que eran entendidos en el arte y la cultura" (Lumbreras 2007:12). La influencia de Tello sobre los museos sólo llegó a la costa y sierra, más no a la Amazonía.

Conforme a los estatutos del Consejo Internacional de Museos (ICOM), organización no gubernamental internacional, fundado en 1946, bajo los auspicios de la UNESCO, la definición de museo en su artículo $2^{\circ}$ es la siguiente: "Un museo es una institución de carácter permanente y no lucrativo al servicio de la sociedad y su desarrollo, abierta al público que exhibe, conserva, investiga, comunica y adquiere, con fines de estudio, educación y disfrute, la evidencia material de la gente y su medio ambiente". Acá el término conservación debe ir entre comillas, es decir "conservación", en el sentido que lo explica Chaumeil (2009), toda vez que la materialización de la cultura es intrínsecamente dinámica.

La necesidad de un Museo Nacional Amazónico se fundamenta en la urgente prioridad de incorporar las sociedades y los ambientes amazónicos al imaginario de la nación peruana. Su aporte coadyuvará también a acercarnos didácticamente al conocimiento diverso de la región, así como a una nueva forma de educación de las nuevas generaciones y formación de los ciudadanos.

Con la aplicación de las políticas cultural y educativa, desde el Museo Nacional Amazónico, podremos ir cambiando algunas percepciones, como el gran desconocimiento de lo que es la Amazonía, su gente y su entorno, por parte de parte nada menos que de los propios ciudadanos y moradores de Iquitos, en el ejemplo más propio nuestro, que siguen viendo a menudo este espacio como un lugar "salvaje", peligroso y atrasado. Llama la atención la imagen de miedo que genera todavía, en las calles de la ciudad de Iquitos, la simple idea de internarse en la selva.

A través del museo, desde un enfoque intercultural, se tendrá pues un nuevo escenario para acercarnos hacia el conocimiento y reconocimiento de la diversidad de pueblos indígenas que aún moran en la Amazonía, sus conocimientos, sus territorios, su bagaje cultural y sus necesidades y anhelos, con lo cual podremos ir encaminándonos hacia el fortalecimiento de las identidades para una mejor convivencia, respetando nuestras diferencias.

El Museo Nacional Amazónico se debe enmarcar en cuatro principios fundamentales: investigación, conservación, difusión y educación (Ravines 1989), ampliados en los campos de las ciencias sociales y ciencias naturales, de manera transversal, con la finalidad de educar al público visitante, fortalecer las identidades culturales y garantizar el respeto hacia las culturas, generar una cultura del saber, generar conciencia sobre la importancia de la protección y respeto de nuestro legado patrimonial cultural (no estático) y natural (también en constante evolución), y generar recursos impulsando las industrias culturales, sin caer en el congelamiento, y consumismo dominante y enajenante que lamentablemente son objeto los pueblos y su materialidad cultural (Chaumeil 2009).

Con la investigación, interdisciplinar, se produce conocimiento, el cual debe coadyuvar a reproducir el saber, y volcarlo hacia la educación, cultura y economía, buscando generar un efecto multiplicador, el mismo que se puede lograr con divulgaciones científicas para una población selecta, y didácticas para un público masivo a través de medios impresos y digital, pero también a través de programas de difusión representados en un espacio de renovable ambiente museográfico.

La infraestructura apropiada en un lugar como un museo permite la adquisición, el préstamo, la conservación preventiva y restauración de los bienes materiales, que pueden ser de índole paleontológica, arqueológica, histórica, etnográfica, contemporánea, artística, y biológica, en sus diversas muestras representativas. Estos bienes conservados, registrados y catalogados garantizan que las actuales generaciones y las futuras venideras puedan conocerlas y valorarlas, pero también puedan investigarlas acorde a los avances de los instrumentales y métodos científicos del momento.

Algunas lenguas amazónicas van desapareciendo, otras tantas ya han desaparecido; algunas culturas amazónicas van perdiendo sus saberes, muchas tantas ya las han perdido; algunas especies de 
flora y fauna van extinguiéndose, otras tantas también ya se extinguieron. Es mucho el camino que seguir para aprender de la inmensidad de conocimientos que encierran nuestra Amazonía, sin embargo poco o nada de lo que nos vienen legando la historia cultural y natural queda registrado de manera tangible con nosotros. Somos testigos de cuántos centros de investigación y museos en el mundo conservan para la posteridad estos testimonios que emergieron de nuestras tierras amazónicas, y que son materia de investigación y exposición, y que hasta ahora nos es ajeno, pues con el Museo Nacional Amazónico podremos ir recuperándolas.

El futuro museo no se limitará a la sola exhibición de piezas, sino sobre todo, de desarrollo de los procesos creativos de las sociedades pasadas y presentes, que incluya un Centro de investigación en ciencias sociales y ambientales, así como talleres abiertos al gran público y a las escuelas donde se desarrollen procesos creativos (música, pintura, danza, baile, escultura, etc.) propios de los pueblos amazónicos, y propuestas alternativas de generación industrias productivas y empleo responsables social y ambientalmente. Así, por ejemplo, con la exposición de una xiloteca de especies maderables amazónicos (que superan las 300 variedades), podemos a la vez dar a conocer a la población la amplitud y riqueza de nuestros bosques, y la necesidad de su conservación, pero también mostrar nuevas alternativas de explotación de los recursos maderables (v.gr.: paliperro o allcocaspi, una madera marrón oscuro con jaspe excepcional, o la caoba que está en peligro de extinción, o maderas amarillas como el quillobordón) sobre la base de la producción con plantones de árboles en áreas desboscadas.

Las piezas y colecciones para ilustrar las exhibiciones -tanto permanentes como temporalesdel futuro museo se podría conseguir a partir de colecciones privadas que se constituyeron en Loreto (por ej. la colección de cerámica del CETA, etc.) y las de la Dirección Regional de Cultura del Ministerio de Cultura (por ej. Quistococha, etc.), así con préstamos de museos peruanos e instituciones (como el Formabiap, etc.) donde existen colecciones amazónicas y de historia natural (UNAP, IIAP).

En este proceso, en el marco del estudio de preinversión para el Museo Nacional Amazónico es clave y muy necesario la inclusión de proyectos investigativos en los campos sociales (arqueología, antropología, historia, lingüística, sociología, arte) y ambientales (biología, forestal, agronomía) aplicados.

En la Amazonía existen más de 200 sitios arqueológicos conocidos pero la cifra fácilmente supera los miles, con su cultura material e inmaterial enterradas, desbarrancándose y destruyéndose; sitios paleontológicos y restos fósiles desconocidos e incógnitos; aproximadamente 1800 Comunidades Nativas inscritas en los registros públicos aunque sabemos que son más, también con su cultura material e inmaterial aún presentes y en proceso de franco retroceso; decenas de pueblos misionales, municipios y veteranas o derruidas iglesias que guardan valiosos documentos históricos carcomidos por el paso del tiempo; aproximadamente 59 lenguas amazónicas sobrevivientes como sus hablantes; miles de ríos, quebradas, caños, cochas, lagos; cientos de suelos; millares de bosques y fauna que la habitan; allí también están el riquísimo reservorio de conocimientos, difusión y aplicación esperando ser investigados.

El Museo Nacional Amazónico, en Iquitos, deberá contar con un espacio amplio, de unas 3 a 5 ha, en cuyo ambiente puedan caber espacios a escala de las especies biológicas representativas de nuestra Amazonía, vivas y colectadas (tallos, raíces, hojas, frutos, etc.), así como especímenes de la cultura material paleontológica (de flora y fauna), arqueológica (cerámica, lítico, piruros, adornos, restos arqueobotánicos, restos arqueofaunísticos, etc.), históricos (época del Caucho, militar), etnográfica (maza, cerbatanas, utensilios de arcilla, vestimentas, etc.), contemporáneo, arte (esculturas, pinturas, cerámicas), literatura, música, cine, como también áreas de laboratorio, de investigación, de conservación, de exposición, de eventos, y de recreación. 
Entre los posibles candidatos para el terreno del museo podrían estar, según las negociaciones:

i. De tres a cinco hectáreas de las 86 ha. del terreno del cuartel militar Vargas Guerra, ubicado a la margen derecha de la Av. El Ejército, al costado de la Plaza Bolognesi.

ii. De tres a cinco hectáreas de las 10 ha del terreno de IVITA de la Universidad Nacional Mayor de San Marcos, ubicado a la margen izquierda de la Av. El Ejército, al costado de los terrenos ocupados actualmente por el Gobierno Regional de Loreto.

iii. De tres a cinco hectáreas de las 57,600 ha del terreno de la Reserva Nacional Alpahuayo-Mishana, a la margen derecha de la carretera Iquitos-Nauta, a 20 kilómetros de la ciudad de Iquitos.

iv. El antiguo local municipal, ubicado en la segunda cuadra de la calle Napo, por la Plaza de Armas de Iquitos.

v. El antiguo hotel Palace, ubicado en la esquina de las calles Putumayo con Malecón Tarapacá, a una cuadra de la Plaza de Armas de Iquitos.

vi.Algún otro terreno propicio para el museo.

El Museo Nacional Amazónico debe ser abierto al público, de todas las edades, estatus y grados educativos. Debería estar adscrito al Sistema Nacional de Museos del Ministerio de Cultura, y su operación y mantenimiento podría estar bajo la administración del Ministerio de Cultura con la participación de un patronato constituido para tal fin; pero también se podría autofinanciar con la participación de organizaciones cooperantes nacionales y extranjeras, y con los ingresos generados por los servicios de tiendas y restaurantes, con actividades de carácter comerciales o de promoción, sin comprometer la calidad de las colecciones ni la atención al público visitante.

\section{BiBLIOGRAFÍA}

ACEITUNO BOCANEGRA, Francisco Javier

2010 "El poblamiento temprano de la floresta húmeda de Colombia: una síntesis regional para un modelo de poblamiento de la cabecera noroccidental de la cuenca del Amazonas". Arqueologia Amazônica 1: 15-33. Edithe Pereira y Vera Guapindaia, organizadoras. Museu Paraense Emilio Goeldi. Instituto do Patrimônio Historico e Artístico Nacional. Secretaria de Estado de Cultura do Pará. Belém, Brasil.

ÁLVAREZ ALONSO, José

2010 “Bosque amazónico: mucho más que árboles". Atlas de comunidades nativas y áreas naturales protegidas del nordeste de la Amazonía peruana, pp. 39-42. Lima: Instituto del Bien Común.

BENAVIDES, Margarita (Editora)

2010 Atlas de comunidades nativas y áreas naturales protegidas del nordeste de la Amazonía peruana. Lima: Instituto del Bien Común. $134 \mathrm{p}$.

BENAVIDES, Margarita

2010 "La Amazonía peruana, territorios indígenas y recursos naturales" Atlas de comunidades nativas y áreas naturales protegidas del nordeste de la Amazonía peruana, pp. 17-34. Lima: Instituto del Bien Común.

CARBAJAL, Fray Gaspar de

2009 Relación del descubrimiento del famoso río grande que desde Su nacimiento hasta el mar descubrió el capitán Orellana en unión de 56 hombres. Iquitos: Editorial Tierra Nueva. 49 p.

CHAUMEIL, Jean Pierre

2009 "El comercio de la cultura. El caso de los pueblos amazónicos". Bulletin del Instituto Francés de Estudios Andinos, 38 (1): 61-74.

CHIRIF TIRADO, Alberto

2010 "Pueblos indígenas del nordeste de la Amazonía peruana, demografía y problemas". Atlas de comunidades nativas y áreas naturales protegidas del nordeste de la Amazonía peruana, pp. 63-64. Lima: Instituto del Bien Común. 
2010 “Áreas naturales protegidas y pueblos indígenas”. Atlas de comunidades nativas y áreas naturales protegidas del nordeste de la Amazonía peruana, pp. 70-79. Lima: Instituto del Bien Común.

DEL ÁGUILA CHÁVEZ, Carlos

2007 “Tello y San Marcos”. Julio C. Tello, pp. 5-6. Lima: Centro Cultural de San Marcos, UNMSM - IPCNA. ESPINOZA SORIANO, Waldemar

2007 Amazonía del Perú. Historia de la gobernación y comandancia general de Maynas. (Hoy regiones de Loreto, San Martín, Ucayali y provincia de Condorcanqui). Del siglo XV a la primera mitad del siglo XIX. Lima: Fondo Editorial del Congreso del Perú, Banco Central de Reserva del Perú, PROMPERÚ. 598 p.

FLORES PAITÁN, Salvador

1998 “Agroforestería amazónica: una alternativa a la agricultura migratoria". En: Kalliola y Flores Paitán, editores, Geoecología y desarrollo Amazónico: estudio integrado en la zona de Iquitos, Perú, pp. 417-440. Turku: Turun Yliopisto.

KALLIOLA Risto y Maarit PUHAKKA

1993 "Geografía de la selva baja peruana". En: Kalliola, Puhakka y Danjoy editores, Amazonía Peruana; vegetación húmeda tropical en el llano subandino. pp. 9-21. Turku: Gummerus Printing.

LUMBRERAS, Luis Guillermo

2007 “Apuntes sobre Julio C. Tello, el Maestro". Julio C. Tello, pp. 7-20. Lima: Centro Cultural de San Marcos, UNMSM - IPCNA.

LEY N² 28296. LEY GENERAL DEL PATRIMONIO CULTURAL DE LA NACIÓN. Ministerio de Cultura.

LEY N² 26834. LEY DE ÁREAS NATURALES PROTEGIDAS. Ministerio del Ambiente.

MARENGO, José A.

1998 “Climatología de la zona de Iquitos, Perú”. En: Kalliola y Flores Paitán, editores, Geoecología y desarrollo amazónico: estudio integrado en la zona de Iquitos, Perú, pp. 35-57. Turku: Turun Yliopisto.

NEVES, Eduardo Góes

2011 "El nacimiento del "Presente Etnográfico": la emergencia del patrón de distribución de sociedades indígenas y familias lingüísticas en la tierras bajas sudamericanas, durante el primer milenio d.C.”. En: Chaumeil, Espinosa de Rivero y Cornejo Chaparro, eds. Por donde hay soplo. Estudios Amazónicos en países andinos, pp. 39-65. Actas \& Memories 29. Lima: Instituto Francés de Estudios Andinos, Fondo Editorial de la Pontificia Universidad Católica del Perú, Centro Amazónico de Antropología y Aplicación Práctica, Centre Enseignement et Recherche en Ethnologie Amérindienne du Laboratoried'Ethnologie et de Sociologie Comparative.

ORDENANZA REGIONAL Nº15-GRL/CR. Gobierno Regional de Loreto.

POZI-ESCOT, Inés

1998 El multilingüismo en el Perú. Biblioteca de la Tradición Oral Andina 17. Cusco: Centro de Estudios Regionales Andinos "Bartolomé de Las Casas", Programa de Formación en Educación Intercultural Bilingüe para los Países Andinos. 310 p.

PULGAR VIDAL, Javier

1985 “Las tres selvas del Antisuyo”. En: Boletín de Lima. Año 7, № 39: 59-72. Lima, Perú.

RAVINES, Roger

1989 Los museos del Perú. Breve historia y guía. Lima: Dirección General de Museos. Instituto Nacional de Cultura.

RIVAS PANDURO, Santiago

2011 "Proyectos culturales de inversión pública para Loreto. Entre la memoria y el olvido". Semanario de actualidades Kanatari, año 27, 30 de octubre de 2011, N 1415: 7482-7484, Tomo XXIX. Iquitos: Centro de Estudios Teológicos de la Amazonía.

RUMRRILL, Róger

2011 "La Amazonía y la nueva utopía social en el Siglo XXI". Libros \& Artes. Revista de la Biblioteca Nacional del Perú, pp. 2-5. Lima, Perú. 
SOLIS FONSECA, Gustavo

2003 Lenguas en la Amazonía Peruana. Lima: Edición por demanda. 246 p.

SOTIL GARCÍA, Gabel Daniel

2002 “Iquitos, ¿hacia dónde vas?". Revista Conmemorativa. 138 aniversario de la fundación del puerto fluvial de Iquitos sobre el río Amazonas, pp. 8-10. Iquitos, Perú.

UNESCO

2005 Directrices prácticas para la aplicación de la Convención del Patrimonio Mundial. Comité Intergubernamental De Protección Del Patrimonio Mundial Cultural Y Natural. París: Centro del Patrimonio Mundial de la UNESCO. Disponible en < http://whc.unesco.org/archive/opguide05-es. pdf>

VEGA, César A.

2008 Amazon River. Iquitos - Loreto - Peru.Turism, Routes and Customs. Gobierno Regional de Loreto / Guías Perú. Lima, Perú. 240 p. 